\title{
Differential item functioning for items in Berger's HIV Stigma Scale: an analysis of cohorts from the Indian, Swedish, and US contexts
}

\author{
Maria Reinius ${ }^{1}$ (1) - Deepa Rao ${ }^{6} \cdot$ Lisa E. Manhart $^{7} \cdot$ Maria Wiklander $^{2} \cdot$ Veronica Svedhem $^{3,5} \cdot$ John Pryor $^{8}$. \\ Randall Mayer ${ }^{9} \cdot$ Bambi Gaddist $^{10} \cdot$ Shuba Kumar $^{12} \cdot$ Rani Mohanraj $^{12} \cdot$ Lakshmanan Jeyaseelan $^{11}$. \\ Lena Wettergren $^{2,13} \cdot$ Lars E. Eriksson ${ }^{1,3,4}$
}

Accepted: 19 March 2018 / Published online: 24 March 2018

(c) The Author(s) 2018

\begin{abstract}
Purpose To examine whether items in Berger's HIV Stigma Scale function differently with persons of different age, gender, and cultural backgrounds.

Methods Secondary data from cohorts, collected in South India $(n=250)$, Sweden $(n=193)$, and the US $(n=603)$ were reanalyzed to evaluate DIF within, between, and across these cohorts. All participants had answered the revised version of the HIV stigma scale consisting of 32 items forming the subscales Personalized stigma, Disclosure concerns, Concerns about public attitudes, and Negative self-image. Differential Item Functioning (DIF) for these items was assessed using hybrid ordinal regression-IRT technique. When DIF was detected, the cumulative impact of DIF on individual subscale scores was evaluated.

Results DIF was detected for 9 items within, between, or across cohorts, but the DIF was negligible in general. Detected DIF between the Swedish and Indian cohorts had a cumulative salient impact on individual scores for the subscale Disclosure Concerns; Disclosure concerns were overestimated in the Swedish cohort and both over- and underestimated in the Indian cohort.

Conclusions The items in the 32-item version of the HIV stigma scale did not seem to be particularly prone to present DIF. The DIF between the Indian and Swedish cohort for items in the subscale Disclosure Concerns could, however, result in both type I and type II errors if scores should be compared between the Indian and Swedish cohort.
\end{abstract}

Keywords HIV · Stigma · The HIV stigma scale · Differential item functioning · Item Response Theory · Psychometrics

\section{Background}

Since the beginning of the pandemic, people with HIV have been stigmatized. Mahajan [1] defines HIV-related stigma as when people living with HIV are labeled, stereotyped, experience separation and status loss, and become discriminated both on an individual and structural level. Across different contexts perceived HIV-related stigma is associated to poor mental and physical health for persons living with HIV [2-5]. Even in the current era of efficient

Electronic supplementary material The online version of this article (https://doi.org/10.1007/s11136-018-1841-4) contains supplementary material, which is available to authorized users.

Maria Reinius

maria.reinius@ki.se

Extended author information available on the last page of the article treatment, making HIV a chronic illness with normal life expectancy where treatment is generally available, people living with HIV are exposed to and relate to HIV-related stigma [6, 7] and stigma has been found to be a common barrier to treatment and prevention [8,9].

Stigma has sometimes been understood as an individual process, where stigma is constituted of what some individuals do to others [10], whereas other scholars have argued that an individual perspective of stigma only may be relevant in highly individualized countries (as the US or some parts of Europe) [11]. Parker and Aggleton [11] argue that stigma, where it appears, is strongly related to the specific context of culture and power, and that the social and cultural phenomenon of stigma may be linked to actions of whole groups of people. Some known aspects of HIV-related stigma are also clearly cultural specific, as they, for example, relate to specific religious beliefs [12]. 
The efforts to reduce HIV-related stigma have yet not matched the magnitude of the problem [13]. Parker and Aggleton suggest that the diversity and complexity of HIV-related stigma makes it difficult to grasp in a programmatically useful way [11]. Ogden and Nyblade, on the other hand, argue that differences in HIV-related stigma across cultures are largely superficial and that stigma is expressed remarkably consistent across contexts [13]. Valid and reliable instruments for measuring HIV-related stigma are essential for stigma research, for evaluation of stigma-reducing interventions, and for monitoring and understanding experiences of HIV-related stigma [10, 14, 15]. Based on findings that suggest consistencies in HIVrelated stigma across cultures [13], it would be valuable to have measures of HIV-related stigma that are valid across cultures, thus enabling measurement of changing trends in HIV-related stigma over time and across contexts.

One of the many instruments designed to measure HIVrelated stigma perceived by persons living with HIV is the HIV Stigma Scale by Berger et al. [16]. Although developed and originally found valid and reliable in a US context [16], the instrument has been translated and found to be relevant and valid across various countries and cultures $[17,18]$. These findings suggest that HIV-related stigma as assessed by the Berger's scale is, to some extent, universal. For example, the HIV Stigma Scale has been used to compare levels of perceived HIV-related stigma among persons living with HIV in Kenyan, Puerto Rican, and the United States contexts [3], where Hispanics reported significantly higher levels of stigma than persons from the African continent. When used in Kenya, Puerto Rico, and the United States, the HIV Stigma Scale showed good internal consistency measured by Cronbach's $\alpha$ for the combined data. The authors, however, did not explore differential item functioning for items in the HIV Stigma Scale, and it is possible that participants from different countries interpreted the items differently, resulting in bias.

Participants' interpretation of scales can be assessed with methods based on Item Response Theory (IRT) and analysis of Differential Item Functioning (DIF). As items in the HIV Stigma Scale are statements that participants are requested to agree or disagree with on a four-point Likert scale, the probability that an individual will agree with the statement on a certain item can, according to IRT, be seen as a mathematical function of how stigmatized the person is and how severe the stigma is that the item captures. Items in the HIV Stigma Scale would be considered to have DIF if participants with different sociodemographic backgrounds have unequal probabilities of agreeing with statements in the items, while experiencing the same level of stigma [19]. For example, an analysis of DIF in an American sample, showed that black, non-Hispanic persons and white, nonHispanic persons with the same level of stigma, had different probabilities of agreeing with items in the HIV Stigma Scale, indicating that persons with different backgrounds may experience HIV-related stigma differently [20].

In addition to its use in several studies in the United States [21-23], the HIV Stigma Scale has been used, for example, in Sweden [18] and South India [17], providing an ideal opportunity to evaluate whether DIF occurs. The HIV Stigma Scale has been translated into the respective native languages, back translated into English, and checked for comparability with the original English questionnaire $[17,18]$. The English, Tamil, and Swedish versions of the instrument could therefore be considered consistent regarding content and all items were found relevant for both a Swedish and a South Indian context by both experts and people living with HIV in each country [17, 18]. There are, however, results from the psychometric validation from both the Swedish and the Indian contexts that may indicate that at least parts of the concept of HIV-related stigma are culturally embedded. For the Swedish version, a high rate of missing responses were found for an item regarding the risk of losing employment if one's HIV status is disclosed, where written comments in the margin of the questionnaire ("Does this happen in Sweden?") indicated that some Swedish respondents found this item irrelevant [18]. In validation of the Indian version of the HIV Stigma Scale, respondents had difficulties understanding the four-point Likert scale and the word "unclean" in the item "Having HIV makes me feel unclean" was often misinterpreted to mean "personal hygiene" [17].

Although the HIV Stigma Scale has been used for measuring stigma in a wide range of different contexts, it is not clear whether items in the HIV Stigma Scale are interpreted differently by persons with different backgrounds. The aim of the present study was, therefore, to examine whether items in the HIV Stigma Scale function differently with regard to gender and cultural background.

\section{Method}

\section{Overview}

The present work builds on secondary analysis of data collected using the HIV Stigma Scale in India [17], Sweden [18], and three areas in the US, South Carolina [24], Iowa [24], and the city of Chicago [20]. Item-level data from these studies were used to evaluate items for DIF between, within, and across the cohorts. A hybrid ordinal logistic regression-IRT approach [25] was used for DIF detection, using the lordif-package [26] with R statistics [27]. When DIF was detected, we evaluated the cumulative impact that the detected DIF had on individual IRT scores [28]. 


\section{Data source}

Data from the Swedish cohort were collected at the Karolinska University hospital in Stockholm in 2013 from 193 persons living with HIV [18]. Data for the South Indian cohort were collected from 2007 to 2008 through networks caring for men and women living with HIV in the state of Tamil Nadu. Participants were residing in and around the cities Chennai $(n=150)$, which is an urban area and Vellore $(n=100)$, a semirural area [17]. Data from the United States were collected at two different time points in three different states. Between 2005 and 2010, data were collected through Ryan White II/III clinics and a community-based organization providing medical case management in South Carolina $(n=210)$ [20], from Iowa Department of Public Health Title II case-managed clients in Iowa $(n=331)$ [20], and a private University Hospital HIV Clinic in Chicago $(n=62)$ [24].

Available data on sociodemographic information differed across location. Information about gender and age was available for all participants from Sweden, India, and Chicago (US). Gender was categorized as male, female, or transgender in data collected in Chicago, with one participant categorized as transgender. For all other data, gender was categorized as male or female. Differences between cohorts were assessed with $\chi^{2}$-test for gender and ANOVA with Tukey post hoc analysis for age.

\section{The HIV Stigma Scale}

The HIV Stigma Scale is designed to measure four dimensions of perceived HIV-related stigma among persons living with HIV: Personalized stigma, Disclosure concerns, Concerns about public attitudes, and Negative self-image. Personalized stigma is proposed to capture perceived consequences of other people knowing about one's HIV infection. Disclosure concerns captures concerns that one can have over disclosing one's HIV status to others. Concerns about public attitudes includes concerns that one can have over what other people think about people with HIV. Negative self-image includes negative feelings that one can have about oneself related to one's HIV, (e.g., feeling unclean or not as good as others because of HIV) [16]. In scale construction of the original HIV Stigma Scale, items were assigned to dimensions following an exploratory factor analysis where a majority of items cross-loaded. Seventeen items were therefore assigned to more than one dimension. Subscales were subsequently refined to consist of unique items in a revised 32-item version [29]. Since DIF analyses with the lordifpackage assume unidimensionality in the latent trait, where all items measure a single concept [19], the items that compose the revised 32-item version of the HIV Stigma Scale were used in the present work. The instrument includes 32 statements related to HIV stigma that are completed on a four-point Likert scale, ranging from 1 ("completely disagree") to 4 ("completely agree"), and item scores for each subscale are summed into subscale scores [16]. Higher scores indicate higher levels of perceived stigma for all four subscales.

\section{Unidimensionality}

Unidimensionality for scales was assessed through confirmatory factor analysis (CFA), where all items in each scale were specified to load onto one single factor, respectively. Since responses to the HIV stigma scale are ordinal, the CFA models were fitted using weighted least squares means and variance (WLSMV) [30]. Measures of model fit used were as follows: Chi-square (expected to be non-significant if the model showed acceptable fit to the data), the Comparative Fit Index and the Tucker-Lewis Index (CFI and TLI, cut-off $>0.95$ ), and the root mean square error of approximation (RMSEA, cut-off $<0.08)[31,32]$. Since Chi-squares were significant in all CFA's but three and RMSEA exceeded the cut-off for two subscales regarding the Swedish cohort, we also assessed the dimensionality of the models exploratory with parallel analysis and the Empirical Kaiser Criterion [33].

\section{DIF detection}

A combined ordinal logistic regression-IRT approach for DIF detection and analysis of DIF impact [25]—was employed, as implemented in the lordif-package [26]. First, IRT item parameter estimates were obtained, using the mirt package [34], according to the graded response model [35]. The graded response model was chosen over the partial credit model since all items had the same number of response categories (four). Missing values were not imputed; participants were excluded listwise in each separate analysis if missing values were present. The minimum cell count for not collapsing response categories was set to five. Three ordinal regression models were examined for each item:

Model 1 (no DIF) : $\operatorname{logit} P\left(u_{\mathrm{i}} \geq k\right)=\alpha_{\mathrm{k}}+\beta_{1} \times \theta$

Model 2 (uniform DIF) : $\operatorname{logit} P\left(u_{\mathrm{i}} \geq k\right)=\alpha_{\mathrm{k}}+\beta_{1} \times \theta+\beta_{2} \times$ group

Model 3 (non - uniform) DIF $: \operatorname{logit} P\left(u_{\mathrm{i}} \geq k\right)$

$$
=\alpha_{\mathrm{k}}+\beta_{1} \times \theta+\beta_{2} \times \text { group }+\beta_{2} \times \theta \times \text { group },
$$

where $P\left(u_{\mathrm{i}} \geq k\right)$ represented probabilities that item response $u_{\mathrm{i}}$ is $k$ or higher [26], $\theta$ was the latent trait in the present work, four dimensions of perceived HIV-related stigma (Personalized stigma, Disclosure Concerns, Concerns of public attitudes and Negative Self-image). Group was a variable indicating group (country or gender) and $(\theta \times$ group) represented the interaction between the latent trait and group. Pseudo $R^{2}$ changes (Nagelkerke) were used as a criterion 
for DIF detection. When pseudo $R^{2}$ changes for an item exceeded the empirical threshold, that item was flagged for DIF. Pseudo $\mathrm{R}^{2}$ changes between Model 1 and Model 2 indicate a uniform DIF, with a constant effect for different levels of stigma while pseudo $R^{2}$ changes between Model 2 and 3 indicate a non-uniform DIF, with an effect that varied conditional on levels of stigma. The procedure was extended for multiple groups in analysis of DIF between cohorts [26]. Empirical thresholds for DIF detection were generated through Monte Carlo simulations of DIF-free samples [26]. The empirical thresholds were set to be above the highest value identified in the Monte Carlo simulation, identical for all items in each separate analysis, but varying between analyses [26]. The Monte Carlo-simulated item-level thresholds are presented in Online Resource 1.

An iterative process was used to prevent false-positive and false-negative results that may occur when many items with DIF are included in analysis [25]. Trait estimates were updated using the items that were not flagged for DIF and used as a new threshold for DIF detection. The logistic regression was then repeated and if different items were flagged for DIF than in the initial calculation, trait estimates were updated again based on the DIF-free samples in the most previous round. This procedure was repeated until the same items were flagged for DIF in two successive rounds [26].

Based on the knowledge that HIV-related stigma can be experienced differently by persons living with HIV from different backgrounds [20], a series of DIF analyses was performed between and within each cohort, comparing groups with different backgrounds. For the US cohort, DIF was examined between Black, non-Hispanic persons and White, non-Hispanic persons (data collected in Chicago excluded), as earlier examined by Rao et al. [20]. The US cohort was then combined with the Swedish and South Indian cohort and DIF was assessed between persons living in Sweden, the US, and South India. Since respondents in the different cohorts differed with respect to gender, we also examined whether the HIV Stigma Scale had DIF between men and women. Transgender was in analysis coded as missing since this category only included one respondent. DIF related to gender was assessed for the three cohorts combined and are referred to as DIF across cohorts.

\section{Magnitude of detected DIF}

There are no minimal clinically important score differences specified for the HIV Stigma Scale to define a salient DIF. We therefore evaluated the individual-level cumulative impact of detected DIF through comparing unadjusted IRT scores (with DIF) with adjusted IRT scores (accounted for DIF). Unadjusted IRT scores were subtracted from adjusted IRT scores and if individual IRT scores were unaffected by
DIF, this difference would be zero [28]. Differences that were equal to or exceeded a small effect size (i.e., 0.20) were considered to be salient [36].

\section{Results}

\section{Demographic characteristics}

Demographic characteristics by cohort are shown in Table 1 . The US sample had a significantly higher proportion of males compared to the Indian sample (66\% vs. $\left.50 \%, \chi^{2}=60.8, p<0.001\right)$. Further, the one-way ANOVA showed a statistical significance between group effect of age $(\mathrm{F}(2,495)=126.7, p<0.001)$ (data on participants' age were not available for Iowa and South Carolina). Post hoc comparisons using the Tukey HSD test indicated that the mean age of Indian respondents (mean $=35, \mathrm{SD}= \pm 6$ ) was significantly lower than the mean age of respondents from the US (mean $=43, \mathrm{SD}= \pm 11)$ and Sweden $($ mean $=49$, $\mathrm{SD}= \pm 12), p<0.001$. Mean age for respondents from the US (mean $=43, \mathrm{SD}= \pm 11$ ) was also significantly lower than the mean age of Swedish respondents (mean $=49$, $\mathrm{SD}= \pm 12), p<0.001$.

\section{Unidimensionality}

The unidimensional models yielded reasonable fit for all data combined and for the South Indian and US cohort separately (Table 2) which suggested suitability for unidimensional IRT analysis. For the Swedish cohort, unidimensionality was not supported in the confirmatory analysis for the scales Disclosure concerns and Concerns with public attitudes. The exploratory analysis using parallel analysis suggested one-factor solution in all analyses. The Empirical Kaiser Criterion, however, suggested two-factor solutions for Disclosure concerns and Negative self-image regarding the South Indian cohort and for Disclosure concerns regarding the Swedish cohort.

\section{Fit of the graded response models}

The lowest number of endorsements for a category in a group was six. Since the minimum cell count was set to five, none of the response categories were collapsed.

\section{DIF detection}

Nine items in the HIV Stigma Scale were flagged for DIF with pseudo $R^{2}$ change exceeding the thresholds generated through Monte Carlo simulations of DIF-free samples (Table 3). Both uniform (with a constant effect for different levels of stigma) and non-uniform DIF findings (with 
Table 1 Demographic characteristics of respondents to the HIV Stigma Scale in South India [11], Sweden [12], and the US [11, 14, 18]

\begin{tabular}{lrll}
\hline Cohort & $n$ & $\begin{array}{l}\text { Gender } \\
\% \text { Female }\end{array}$ & $\begin{array}{l}\text { Age }^{\mathrm{b}} \\
\text { Mean, range (SD) }\end{array}$ \\
\hline South India & 250 & $50^{\mathrm{a}}$ & $35,18-49(6)^{\mathrm{c}, \mathrm{d}}$ \\
Sweden & 188 & 43 & $49,19-83(12)^{\mathrm{c}, \mathrm{e}}$ \\
The United States & 598 & $34^{\mathrm{a}}$ & $43,23-68(11)^{\mathrm{d}, \mathrm{e}, \mathrm{f}}$ \\
All & 1036 & 40 & $41,18-83(11)$ \\
\hline
\end{tabular}

Respondents with missing values in one or more of the HIV stigma subscales were excluded

${ }^{a}$ Significant difference between India and the United States $\left(\chi^{2}=60.8\right.$, $p<0.001$ )

${ }^{\mathrm{b}}$ Overall significant difference (ANOVA): $\mathrm{F}(2,495)=126.7, p<0.001$

${ }^{\mathrm{c}}$ Significant difference between India and Sweden, Tukey post hoc test, $p<0.001$

${ }^{\mathrm{d}}$ Significant difference between India and Chicago, Tukey post hoc test, $p<0.001$

${ }^{\mathrm{e}}$ Significant difference between Chicago and Sweden, Tukey post hoc test, $p<0.001$

${ }^{\mathrm{f}}$ Age only available for respondents from Chicago

an effect that varies depending on levels of stigma) were detected.

\section{DIF within cohorts-the US cohort}

For the US cohort, DIF was examined between Black, nonHispanic persons and White, non-Hispanic persons. No items were flagged for DIF within the US cohort.

\section{DIF between cohorts}

Nine items in the HIV Stigma Scale were flagged for either uniform, non-uniform, or both uniform and non-uniform DIF between persons living in Sweden, the US, and South India, respectively. One item for Personalized stigma, five items for Disclosure concerns, one item for Concerns about public attitudes, and two items for Negative self-image (Table 3).

\section{DIF across cohorts—gender}

One item was found to have non-uniform DIF between men and women (Table 3) (1. In many areas of my life, no one knows that I have HIV, belonging to the subscale Disclosure concerns).

\section{Magnitude of detected DIF}

The detected DIF was, in general, negligible, since the cumulative impact of DIF on individual IRT scores was below 0.2. Salient individual-level impacts of DIF (exceeding 0.2) were, however, detected between cohorts for the subscales Disclosure concerns, which are detailed below and shown in Fig. 1.

\section{DIF between cohorts-cumulative DIF impact on individual scores}

Regarding DIF between cohorts, changes in individual IRT scores exceeded 0.2 and were therefore thought to be salient for the subscale Disclosure concerns (Fig. 1). Scores for respondents from the US were mainly unaffected by DIF, but DIF had a salient impact on scores for Indian and Swedish respondents, with effects in opposite directions. For Disclosure concerns, the HIV Stigma Scale seemed to overestimate scores for Swedish participants and both overestimate (for persons with low levels of Disclosure concerns) and underestimate (for persons with high levels of Disclosure concerns) scores for Indian participants (Fig. 1). The mean of the differences was close to zero, indicating that this DIF would not affect the mean score if levels of Disclosure concerns would be presented for persons from the Swedish, US, and South Indian cohort together.

\section{Discussion}

The HIV Stigma Scale was developed for quantitative measurement of HIV-related stigma, as perceived by persons living with HIV. Though adapted and used in diverse contexts, this is the first time that the instrument has been assessed for gender-related DIF and DIF across different cultural contexts. The results of the present study indicate that the items in the HIV stigma scale were not especially prone to present culture-related DIF for the subscales Personalized stigma, Concerns about public attitudes, and Negative self-image. These subscales seem to cover aspects of stigma that in general are equally interpreted regarding content across the different groups investigated. These results indicate that levels of Personalized stigma, Concerns about public attitudes, and Negative self-image may be compared between the cohorts without the risk of results being culturally biased. Salient DIF was, however, found between the South Indian and Swedish cohorts for the subscale Disclosure concerns. The subscale Disclosure concerns measures concerns that one can have over disclosing one's HIV status to others. The items that present DIF mainly covers the aspect that one's HIV is a secret and we can only speculate about potential reasons for this DIF. Since India is more densely populated than Sweden, it may be likely that participants in the South Indian cohort live physically closer to family and neighbors than the participants in the Swedish cohort, making it harder 
Table 2 Assessment of unidimensionality for all subscales in the 32-item HIV Stigma Scale

\begin{tabular}{|c|c|c|c|c|}
\hline \multirow[t]{2}{*}{ Subscale } & \multicolumn{4}{|c|}{ Fit measures from $\mathrm{CFA}^{\mathrm{a}}$} \\
\hline & All data combined & Indian cohort & Swedish cohort & US cohort \\
\hline \multicolumn{5}{|l|}{ Personalized stigma } \\
\hline$n$ & 960 & 244 & 157 & 559 \\
\hline Chi-square $(\mathrm{df}=44)$ & $148.37 *$ & $75.0544 *$ & $67.12 *$ & $150.40 *$ \\
\hline $\mathrm{CFI}$ & 0.998 & 0.995 & 0.999 & 0.998 \\
\hline TLI & 0.998 & 0.993 & 0.999 & 0.997 \\
\hline RMSEA & 0.050 & 0.054 & 0.058 & 0.066 \\
\hline \multicolumn{5}{|l|}{ Disclosure concerns } \\
\hline$n$ & 992 & 250 & 168 & 574 \\
\hline Chi-square $(\mathrm{df}=20)$ & $134.66^{*}$ & $24.74, \mathrm{~ns}$ & $51.87 *$ & $90.50^{*}$ \\
\hline CFI & 0.991 & 0.996 & 0.992 & 0.992 \\
\hline TLI & 0.997 & 0.994 & 0.989 & 0.989 \\
\hline RMSEA & 0.076 & 0.031 & 0.098 & 0.078 \\
\hline \multicolumn{5}{|l|}{ Concerns about public attitudes } \\
\hline$n$ & 965 & 246 & 159 & 560 \\
\hline Chi-square $(\mathrm{df}=9)$ & $39.48^{*}$ & $4.38, \mathrm{~ns}$ & $38.38 *$ & $34.63^{*}$ \\
\hline CFI & 0.997 & 1.000 & 0.987 & 0.997 \\
\hline TLI & 0.995 & 1.008 & 0.979 & 0.995 \\
\hline RMSEA & 0.059 & $<0.001$ & 0.114 & 0.071 \\
\hline \multicolumn{5}{|l|}{ Negative self-image } \\
\hline$n$ & 1010 & 248 & 180 & 582 \\
\hline Chi-square $(\mathrm{df}=14)$ & $45.65^{*}$ & $35.79^{*}$ & $9.72, \mathrm{~ns}$ & $38.28^{*}$ \\
\hline CFI & 0.997 & 0.981 & 1.000 & 0.996 \\
\hline TLI & 0.995 & 0.972 & 1.003 & 0.994 \\
\hline RMSEA & 0.047 & 0.079 & $<0.001$ & 0.055 \\
\hline & \multicolumn{4}{|c|}{ Number of factors proposed to retain by $\mathrm{EKC}^{\mathrm{b}}$} \\
\hline Personalized stigma & 1 & 1 & 1 & 1 \\
\hline Disclosure concerns & 1 & 2 & 2 & 1 \\
\hline Concerns about public attitudes & 1 & 1 & 1 & 1 \\
\hline Negative self-image & 1 & 2 & 1 & 1 \\
\hline
\end{tabular}

Figures in bold indicate that the subscale was not unidimensional according to EKC

${ }^{*} p<0.001$

${ }^{\text {a } C o n f i r m a t o r y ~ f a c t o r ~ a n a l y s i s ~ o f ~ u n i d i m e n s i o n a l ~ m o d e l s, ~ f i t t e d ~ u s i n g ~ t h e ~ w e i g h t e d ~ l e a s t ~ s q u a r e s ~ m e a n ~ a n d ~ v a r i a n c e ~(W L S M V) ~}$

${ }^{\mathrm{b}}$ The Empirical Kaiser Criterion

for the South Indian participants to keep their HIV a secret. India has been characterized as a 'collectivist' society [37] and Sweden more of an individualistic society [38], particularly around interpersonal issues. According to Chadda and Deb [37] family is far more involved in the care of its members in the Indian society, compared to western societies. This might also make it more difficult for the South Indian participants to keep their HIV a secret from their family, compared to the Swedish participants. If keeping one's HIV a secret is difficult, on the edge of being impossible, in the South Indian context, items like "I work hard to keep my HIV a secret" might have been perceived as irrelevant for South Indian participant, thus generating the detected DIF.
When Rao et al. [20] examined DIF between Black, nonHispanic persons and White, non-Hispanic persons, nine items of the scale demonstrated DIF. Our present results did not replicate these findings, as no items were flagged for DIF between Black, non-Hispanic persons and White, nonHispanic persons. A possible explanation for the differences in results could be that Rao et al. [20] examined the 40-item version of the HIV Stigma Scale, while the 32-item version was examined in the present study. Seven of the items, from the 40-item full version of the instrument, that demonstrated DIF in the earlier study by Rao et al. were items that crossloaded in an exploratory factor analysis and therefore were excluded from the 32-item version of the instrument [29]. 
Table 3 Differential item functioning findings for items in Berger's HIV Stigma Scale (32-item version) within, between, and across the three cohorts

\begin{tabular}{|c|c|c|c|c|c|c|}
\hline \multirow[t]{2}{*}{ Items } & \multicolumn{2}{|c|}{$\begin{array}{l}\text { DIF within cohorts } \\
\text { U.S. cohort }{ }^{\mathrm{a}}\end{array}$} & \multicolumn{2}{|c|}{ DIF between cohorts ${ }^{b}$} & \multicolumn{2}{|c|}{$\begin{array}{l}\text { DIF across cohorts } \\
\text { DIF related to gender }\end{array}$} \\
\hline & Uniform DIF & Non-uniform DIF & Uniform DIF & Non-uniform DIF & Uniform DIF & Non-uniform DIF \\
\hline \multicolumn{7}{|l|}{ Personalized stigma $^{\mathrm{d}}$} \\
\hline Threshold $^{\mathrm{h}}$ & \multicolumn{2}{|c|}{0.01} & \multicolumn{2}{|c|}{0.01} & \multicolumn{2}{|c|}{0.01} \\
\hline $\begin{array}{l}\text { 18. Some people } \\
\text { who know I } \\
\text { have HIV have } \\
\text { grown more } \\
\text { distant }\end{array}$ & 0.0002 & 0.0002 & 0.0007 & 0.0029 & 0.0031 & $<0.0001$ \\
\hline $\begin{array}{l}\text { 24. I have been } \\
\text { hurt by how } \\
\text { people reacted } \\
\text { to learning I } \\
\text { have HIV }\end{array}$ & 0.0003 & 0.0004 & 0.0061 & 0.0005 & 0.0079 & $<0.0001$ \\
\hline $\begin{array}{l}\text { 26. I regret hav- } \\
\text { ing told some } \\
\text { people that I } \\
\text { have HIV }\end{array}$ & 0.0016 & 0.0022 & 0.0046 & 0.0003 & 0.0003 & $<0.0001$ \\
\hline $\begin{array}{l}\text { 28. Some people } \\
\text { avoid touching } \\
\text { me once they } \\
\text { know I have } \\
\text { HIV }\end{array}$ & 0.0003 & 0.0000 & 0.0077 & 0.0007 & 0.0001 & $<0.0001$ \\
\hline $\begin{array}{l}\text { 29. People I care } \\
\text { about stopped } \\
\text { calling after } \\
\text { learning I have } \\
\text { HIV }\end{array}$ & 0.0012 & 0.0011 & 0.0016 & 0.0043 & 0.0002 & $<0.0001$ \\
\hline $\begin{array}{l}\text { 32. People don't } \\
\text { want me around } \\
\text { their children } \\
\text { once they know } \\
\text { I have HIV }\end{array}$ & 0.0001 & 0.0000 & 0.0035 & 0.0005 & 0.0001 & $<0.0001$ \\
\hline $\begin{array}{l}\text { 33. People have } \\
\text { physically } \\
\text { backed away } \\
\text { from me when } \\
\text { they learn I } \\
\text { have HIV }\end{array}$ & 0.0000 & 0.0009 & 0.0008 & 0.0018 & 0.0000 & $<0.0001$ \\
\hline $\begin{array}{l}\text { 35. I have stopped } \\
\text { socializing with } \\
\text { some people } \\
\text { because of their } \\
\text { reactions to my } \\
\text { having HIV }\end{array}$ & 0.0005 & 0.0002 & 0.0002 & 0.0000 & 0.0000 & $<0.0001$ \\
\hline $\begin{array}{l}\text { 36. I have lost } \\
\text { friends by tell- } \\
\text { ing them I have } \\
\text { HIV }\end{array}$ & 0.0018 & 0.0014 & 0.0004 & 0.0016 & 0.0012 & $<0.0001$ \\
\hline $\begin{array}{l}\text { 38. People who } \\
\text { know I have } \\
\text { HIV tend to } \\
\text { ignore my good } \\
\text { points }\end{array}$ & 0.0015 & 0.0004 & $0.0115^{\mathrm{h}}$ & 0.0038 & 0.0000 & $<0.0001$ \\
\hline
\end{tabular}


Table 3 (continued)

\begin{tabular}{|c|c|c|c|c|c|c|}
\hline \multirow[t]{2}{*}{ Items } & \multicolumn{2}{|c|}{$\begin{array}{l}\text { DIF within cohorts } \\
\text { U.S. cohort }{ }^{\mathrm{a}}\end{array}$} & \multicolumn{2}{|c|}{ DIF between cohorts ${ }^{b}$} & \multicolumn{2}{|c|}{$\begin{array}{l}\text { DIF across cohorts } \\
\text { DIF related to gender }\end{array}$} \\
\hline & Uniform DIF & Non-uniform DIF & Uniform DIF & Non-uniform DIF & Uniform DIF & Non-uniform DIF \\
\hline $\begin{array}{l}\text { 39. People seem } \\
\text { afraid of me } \\
\text { once they learn } \\
\text { I have HIV }\end{array}$ & 0.0009 & 0.0014 & 0.0004 & 0.0002 & 0.0000 & $<0.0001$ \\
\hline \multicolumn{7}{|l|}{ Disclosure concerns ${ }^{\mathrm{e}}$} \\
\hline Threshold $^{\mathrm{h}}$ & \multicolumn{2}{|c|}{0.02} & \multicolumn{2}{|c|}{0.01} & \multicolumn{2}{|c|}{0.01} \\
\hline $\begin{array}{l}\text { 1. In many areas } \\
\text { of my life, no } \\
\text { one knows that } \\
\text { I have HIV }\end{array}$ & 0.0048 & 0.0007 & $\mathbf{0 . 0 2 9 3}^{\mathrm{i}}$ & $0.0204^{i}$ & $0.0138^{\mathrm{h}}$ & 0.0001 \\
\hline $\begin{array}{l}\text { 4. Telling some- } \\
\text { one I have HIV } \\
\text { is risky }\end{array}$ & 0.0015 & 0.0000 & 0.0005 & 0.0004 & 0.0000 & 0.0011 \\
\hline $\begin{array}{l}\text { 6. I work hard to } \\
\text { keep my HIV a } \\
\text { secret }\end{array}$ & 0.0127 & 0.0010 & $\mathbf{0 . 0 5 1 7 ^ { i }}$ & 0.0061 & 0.0011 & 0.0002 \\
\hline $\begin{array}{l}\text { 17. I am very } \\
\text { careful who I } \\
\text { tell that I have } \\
\text { HIV }\end{array}$ & 0.0000 & 0.0093 & $0.0219^{i}$ & $0.0181^{i}$ & 0.0020 & 0.0000 \\
\hline $\begin{array}{l}\text { 21. I never feel } \\
\text { the need to hide } \\
\text { the fact that I } \\
\text { have HIV (R) }\end{array}$ & 0.0090 & 0.0049 & $0.0242^{i}$ & 0.0009 & 0.0001 & 0.0025 \\
\hline $\begin{array}{l}\text { 22. I worry that } \\
\text { people may } \\
\text { judge me when } \\
\text { they learn I } \\
\text { have HIV }\end{array}$ & 0.0057 & 0.0013 & 0.0023 & 0.0083 & 0.0002 & 0.0000 \\
\hline $\begin{array}{l}\text { 25. I worry that } \\
\text { people who } \\
\text { know I have } \\
\text { HIV will tell } \\
\text { others }\end{array}$ & 0.0000 & 0.0039 & 0.0015 & 0.0012 & 0.0003 & 0.0013 \\
\hline $\begin{array}{l}\text { 37. I have told } \\
\text { people close to } \\
\text { me to keep the } \\
\text { fact that I have } \\
\text { HIV a secret }\end{array}$ & 0.0014 & 0.0004 & 0.0025 & $\mathbf{0 . 0 1 9 1}^{\mathrm{i}}$ & 0.0010 & 0.0011 \\
\hline \multicolumn{7}{|c|}{ Concerns about public attitudes ${ }^{\mathrm{f}}$} \\
\hline Threshold $^{\text {h }}$ & & & & & & \\
\hline $\begin{array}{l}\text { 5. People with } \\
\text { HIV lose their } \\
\text { jobs when their } \\
\text { employers find } \\
\text { out }\end{array}$ & 0.0090 & 0.0006 & $0.0188^{\mathrm{h}}$ & 0.0033 & $<0.0001$ & 0.0015 \\
\hline $\begin{array}{l}\text { 9. People with } \\
\text { HIV are treated } \\
\text { like outcasts }\end{array}$ & 0.0024 & 0.0019 & 0.0070 & 0.0066 & $<0.0001$ & 0.0003 \\
\hline $\begin{array}{l}\text { 10. Most people } \\
\text { believe that a } \\
\text { person who has } \\
\text { HIV is dirty }\end{array}$ & 0.0001 & 0.0014 & 0.0019 & 0.0004 & $<0.0001$ & 0.0004 \\
\hline
\end{tabular}


Table 3 (continued)

\begin{tabular}{|c|c|c|c|c|c|c|}
\hline \multirow[t]{2}{*}{ Items } & \multicolumn{2}{|c|}{$\begin{array}{l}\text { DIF within cohorts } \\
\text { U.S. cohort }{ }^{\mathrm{a}}\end{array}$} & \multicolumn{2}{|c|}{ DIF between cohorts ${ }^{b}$} & \multicolumn{2}{|c|}{$\begin{array}{l}\text { DIF across cohorts } \\
\text { DIF related to gender }\end{array}$} \\
\hline & Uniform DIF & Non-uniform DIF & Uniform DIF & Non-uniform DIF & Uniform DIF & Non-uniform DIF \\
\hline $\begin{array}{l}\text { 14. Most people } \\
\text { think that a per- } \\
\text { son with HIV is } \\
\text { disgusting }\end{array}$ & 0.0004 & 0.0005 & 0.0061 & 0.0006 & $<0.0001$ & 0.0000 \\
\hline $\begin{array}{l}\text { 16. Most people } \\
\text { with HIV are } \\
\text { rejected when } \\
\text { others find out }\end{array}$ & 0.0034 & 0.0001 & 0.0017 & 0.0038 & $<0.0001$ & 0.0001 \\
\hline $\begin{array}{l}\text { 20. Most people } \\
\text { are uncomfort- } \\
\text { able around } \\
\text { someone with } \\
\text { HIV }\end{array}$ & 0.0000 & 0.0048 & 0.0008 & 0.0034 & $<0.0001$ & 0.0000 \\
\hline \multicolumn{7}{|l|}{ Negative self-image ${ }^{\mathrm{g}}$} \\
\hline Threshold $^{\mathrm{h}}$ & \multicolumn{2}{|c|}{0.02} & \multicolumn{2}{|c|}{0.01} & \multicolumn{2}{|c|}{0.01} \\
\hline $\begin{array}{l}\text { 2. I feel guilty } \\
\text { because I have } \\
\text { HIV }\end{array}$ & 0.0032 & 0.0002 & 0.0006 & 0.0048 & 0.0003 & 0.0000 \\
\hline $\begin{array}{l}\text { 3. People's } \\
\text { attitudes about } \\
\text { HIV make me } \\
\text { feel worse about } \\
\text { myself }\end{array}$ & 0.0005 & 0.0000 & $0.0174^{\mathrm{h}}$ & 0.0010 & 0.0021 & 0.0006 \\
\hline $\begin{array}{l}\text { 7. I feel I am } \\
\text { not as good a } \\
\text { person as others } \\
\text { because I have } \\
\text { HIV }\end{array}$ & 0.0000 & 0.0000 & 0.0026 & 0.0005 & 0.0001 & 0.0009 \\
\hline $\begin{array}{l}\text { 8. I never feel } \\
\text { ashamed of hav- } \\
\text { ing HIV (R) }\end{array}$ & 0.0001 & 0.0064 & $0.0502^{\mathrm{h}}$ & 0.0056 & 0.0002 & 0.0037 \\
\hline $\begin{array}{l}\text { 12. Having HIV } \\
\text { makes me feel } \\
\text { unclean }\end{array}$ & 0.0015 & 0.0002 & 0.0044 & 0.0004 & 0.0003 & 0.0000 \\
\hline $\begin{array}{l}\text { 15. Having HIV } \\
\text { makes me feel } \\
\text { like I'm a bad } \\
\text { person }\end{array}$ & 0.0013 & 0.0004 & 0.0044 & 0.0007 & 0.0001 & 0.0008 \\
\hline $\begin{array}{l}\text { 23. Having HIV } \\
\text { in my body is } \\
\text { disgusting to } \\
\text { me }\end{array}$ & 0.0004 & 0.0003 & 0.0045 & 0.0032 & 0.0021 & 0.0010 \\
\hline
\end{tabular}

Empirical thresholds for DIF detection were generated through Monte Carlo simulations of DIF-free samples

${ }^{a}$ DIF between White non-Hispanics and Black non-Hispanics (data collected in Chicago excluded)

${ }^{b}$ DIF between persons living in Sweden, US, and South India

${ }^{c}$ DIF between men and women (transgender coded as missing)

d290 white non-hispanic and 207 Black non-Hispanic participants in within cohort analysis; 559 US, 244 South Indian, and 157 Swedish participants in between cohort analysis; 586 male and 371 female participants in analysis across cohorts

e 303 White non-Hispanic and 209 Black non-Hispanic participants in within cohort analysis; 574 US, 250 South Indian, and 168 Swedish participants in between cohort analysis; 599 male and 390 female participants in analysis across cohorts

f 296 White non-Hispanic and 206 Black non-Hispanic participants in within cohort analysis; 560 US, 246 South Indian, and 159 Swedish participants in between cohort analysis; 583 male and 379 female participants in analysis across cohorts 
Table 3 (continued)

g308 White non-Hispanic and 212 Black non-Hispanic participants in within cohort analysis; 582 US, 248 South Indian, and 180 Swedish participants in between cohort analysis; 606 male and 401 female participants in analysis across cohorts

${ }^{\text {h}}$ Italic values indicate pseudo $R^{2}$ change for items that were flagged for DIF, but where DIF did not have a salient impact on individual scores. The threshold for DIF detection was generated through Monte Carlo simulations of DIF-free items

${ }^{\mathrm{i}}$ Bold values indicate items that were flagged for DIF and where DIF had a cumulative salient impact on individual IRT scores

Fig. 1 Cumulative individuallevel DIF impact for subscale Disclosure concerns for DIF between persons living in South India (triangles), Sweden (plus signs), and the US (circles). The boxplot to the left shows differences in IRT score between using scores that ignore DIF and scores that account for DIF. In the scatterplot to the right, the difference scores (initialpurified) are plotted against the initial scores ignoring DIF (initial theta)

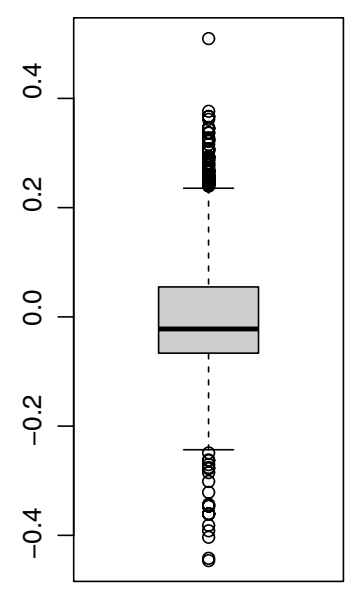

A common reason for DIF is a lack of translation equivalence [19]. The item "Having HIV makes me feel unclean" (Item 12), for example, could not be adequately translated to Tamil, since no words in Tamil captured the intended meaning of "unclean" [17]. This was adjusted for by letting the questionnaire be administered by professional raters in the South Indian cohort, who assured that item content was understood as intended [17]. A similar procedure was used for the Swedish cohort where members of the research team also were present to answer questions and assist respondents with explanations of items if needed [18]. The item "Having HIV makes me feel unclean" (Item 12) did not demonstrate DIF in the present analysis, but if the HIV Stigma Scale is used as a self-administered instrument as originally intended, the detected DIF may possibly be even more pronounced.

Unidimensionality was assessed with both confirmatory and exploratory methods. Since confirmatory methods indicated that some subscales might not be unidimensional, dimensionality was examined further with both parallel analysis and the Empirical Kaiser Criterion. Parallel analysis [39] supported unidimensionality for all subscales across all cohorts, while the Empirical Kaiser Criterion suggested two-factor solutions for the South Indian and Swedish cohort. Parallel analysis is an often recommended approach for dimensionality assessment [39]. The HIV stigma scale is, however, an instrument constituted of oblique, highly

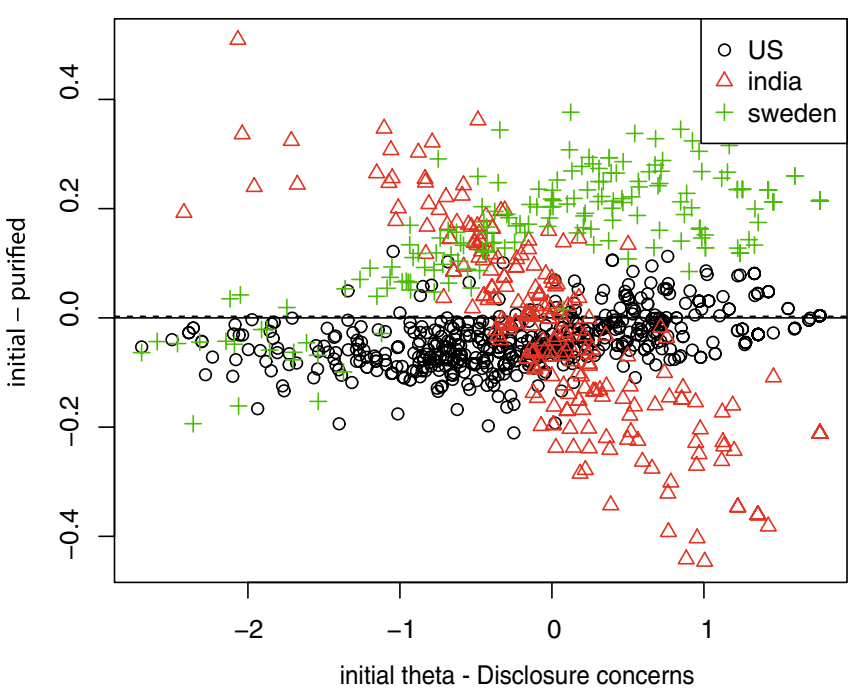

correlated factors [16, 18], and for this specific case the Empirical Kaiser Criterion has been shown to outperform parallel analysis [33]. We therefor conclude that the subscales Disclosure concerns and Negative self-image may be, at least, bi-factorial scales when used in the Swedish or South Indian context. These findings may have implications for the interpretation of the detected DIF, as multidimensionality can be mistaken for DIF [40].

There are several techniques available for DIF detection, and there is a lack of consensus regarding thresholds for detection of DIF [28]. As we used a hybrid ordinal logistic regression-IRT approach, other techniques may have produced different results. Since statistical significance not necessarily implies practical significance, we used a measure of effect size (changes in pseudo $R^{2}$ ) as a criterion for when DIF should be detected [19]. Different sets of rules have been presented for when pseudo $R^{2}$ should be considered to represent DIF [19], where Zumbo [41] suggested that cut-offs indicating moderate and large DIF should be 0.13 and 0.26 , respectively, while the Jodoin and Gierl approach suggests cut-offs of 0.035 and 0.070 [42]. A cutoff level of 0.02 has also been commonly used and these different cut-offs can, unsurprisingly, produce very different numbers of items flagged for DIF [19]. In the present work we used Monte Carlo simulations, as implemented in the lordif-package [26], to generate empirical cut-offs. This rendered cut-offs as low as 0.01 for some analyses, 
which also resulted in detection of DIF that did not have a salient impact on individual scores. This low threshold for DIF detection was, however, set knowing that cultural DIF probably was adjusted for already in data collection and we sought to find patterns in present DIF that perhaps would have been more pronounced if questionnaires had been exclusively self-administered.

Limitations in the present work are that data were collected at different time points with over 5 years apart and that data from the United States and India were not collected with an intention to be representative for people living with HIV in the respective countries [17, 20, 24]. In those settings, the study populations represented a sub-set of all people living with HIV. The results, therefore, cannot be generalized to represent differences between countries. Furthermore, we do not know if the results would be replicated if data were collected at the same locations today. A recommended minimum sample size for ordinal logistic regression is 200 participants per group [19]. Thus, a limitation in the present study is the Swedish sample size of 157-180 participants depending on subscale analyzed. In a simulation study, sample sizes as low as 100 per group were sufficient to detect large DIF but not moderate DIF [43]. It is therefore possible that the present study failed to detect moderate but practically important DIF. Further limitations were that the datasets differed regarding gender and age, and we did not have access to other sociodemographic data. It is generally known that HIV-related stigma is linked to stigma related to other attributes, which can potentiate the power of stigmatization [1]; groups of people who are already exposed of racism, homophobia, sexism, or poverty are predisposed to greater HIV-related stigma [1]. As we did not have access to data other than ratings to the HIV stigma scale and gender and, to some extent, age, we were not able to control for potentially confounding variables that may have been a true source of DIF. For the HIV Stigma Scale it would also be interesting to analyze if DIF occurs between groups of different health statuses, e.g., persons who are virally suppressed or not, persons who have been living with HIV for a longer or shorter period of time.

Aside from these limitations, we propose that the results in the present work indicate that the items in the subscales Personalized stigma, Concerns about public attitudes, and Negative self-image in the HIV Stigma Scale are not especially prone to present salient DIF. However, the detected DIF between the Indian and Swedish cohort for the subscale Disclosure concerns did have a cumulative, albeit small, salient effect on individual IRT scores, which could result in both type I and type II errors if levels of Disclosure concerns should be compared between the Swedish and Indian cohorts. The detected DIF in the subscale Disclosure concerns could be a "true" differential item functioning, i.e., that persons from the Swedish and South Indian cohort respond differently to items regarding "keeping one's HIV a secret" and "hiding one's HIV," respectively, even after accounting for their overall level of disclosure concerns. One possible explanation for this could be differences in the actual possibility to keep private things a secret in the Indian society, compared to the Swedish. An additional possible explanation could be that the unidimensional nature that the subscale Disclosure concerns seems to have in the Swedish and South Indian cohort may have caused a DIF due to multidimensionality. Apart from the cause of the detected DIF, we cannot recommend the subscales Disclosure concerns for comparisons between Sweden and South India.

The results in the present study, however, support the use of the subscales Personalized stigma, Concerns about public attitudes, and Negative self-image for comparisons of levels of stigma between the cohorts investigated. As the HIV Stigma Scale is being used to assess stigma in a wide range of different countries, we encourage researchers using the HIV Stigma Scale to cooperate across country borders and examine the cross-cultural validity of the instrument further. This would broaden the understanding of the extent and forms of stigma faced by people living with HIV in different countries.

Acknowledgements The work presented here was supported by grants from the Doctoral School in Health Care Sciences, Karolinska Institutet.

\section{Compliance with ethical standards}

Conflict of interest The authors declare that they have no conflict of interests.

Ethical approval All procedures performed were in accordance with the ethical standards of the institutional and/or national research committee and with the 1964 Helsinki Declaration and its later amendments or comparable ethical standards. Procedures for data collection were approved by the Regional Ethical Review Board of Stockholm for data collection in Sweden, by the Institutional Review Boards of the Christian Medical College, Vellore, and the University of Washington for data collection in India, and by Northwestern University and Illinois State University for data collections in the US. Informed consent was obtained from all individual participants included in the study.

Open Access This article is distributed under the terms of the Creative Commons Attribution 4.0 International License (http://creativeco mmons.org/licenses/by/4.0/), which permits unrestricted use, distribution, and reproduction in any medium, provided you give appropriate credit to the original author(s) and the source, provide a link to the Creative Commons license, and indicate if changes were made.

\section{References}

1. Mahajan, A. P., Sayles, J. N., Patel, V. A., Remien, R. H., Sawires, S. R., Ortiz, D. J., et al. (2008). Stigma in the HIV/ 
AIDS epidemic: A review of the literature and recommendations for the way forward. AIDS, 22(Suppl 2), S67-S79. https://doi. org/10.1097/01.aids.0000327438.13291.62.

2. Logie, C., \& Gadalla, T. M. (2009). Meta-analysis of health and demographic correlates of stigma towards people living with HIV. AIDS Care, 21(6), 742-753. https://doi.org/10.1080/0954012080 2511877.

3. Holzemer, W. L., Human, S., Arudo, J., Rosa, M. E., Hamilton, M. J., Corless, I., et al. (2009). Exploring HIV stigma and quality of life for persons living with HIV infection. Journal of the Association of Nurses in AIDS Care, 20(3), 161-168. https://doi. org/10.1016/j.jana.2009.02.002.

4. Slater, L. Z., Moneyham, L., Vance, D. E., Raper, J. L., Mugavero, M. J., \& Childs, G. (2013). Support, stigma, health, coping, and quality of life in older gay men with HIV. Journal of the Association of Nurses in AIDS Care, 24(1), 38-49. https://doi. org/10.1016/j.jana.2012.02.006.

5. Wu, X., Chen, J., Huang, H., Liu, Z., Li, X., \& Wang, H. (2014). Perceived stigma, medical social support and quality of life among people living with HIV/AIDS in Hunan, China. Applied Nursing Research. https://doi.org/10.1016/j.apnr.2014.09.011.

6. Walker, L. (2017). 'There's no pill to help you deal with the guilt and shame': Contemporary experiences of HIV in the United Kingdom. Health (London). https://doi.org/10.1177/1363459317 739436.

7. Persson, A. (2013). Non/infectious corporealities: Tensions in the biomedical era of 'HIV normalisation'. Sociology of Health and Illness, 35(7), 1065-1079. https://doi.org/10.1111/14679566.12023.

8. Sweeney, S. M., \& Vanable, P. A. (2016). The association of HIV-related stigma to HIV medication adherence: A systematic review and synthesis of the literature. AIDS and Behavior, 20(1), 29-50. https://doi.org/10.1007/s10461-015-1164-1.

9. Langebeek, N., Gisolf, E. H., Reiss, P., Vervoort, S. C., Hafsteinsdottir, T. B., Richter, C., et al. (2014). Predictors and correlates of adherence to combination antiretroviral therapy (ART) for chronic HIV infection: a meta-analysis. BMC Medicine. https://doi.org/10.1186/s12916-014-0142-1.

10. Earnshaw, V. A., \& Chaudoir, S. R. (2009). From conceptualizing to measuring HIV stigma: A review of HIV stigma mechanism measures. AIDS and Behavior, 13(6), 1160-1177. https:// doi.org/10.1007/s10461-009-9593-3.

11. Parker, R., \& Aggleton, P. (2003). HIV and AIDS-related stigma and discrimination: A conceptual framework and implications for action. Social Science \& Medicine, 57(1), 13-24.

12. Steward, W. T., Herek, G. M., Ramakrishna, J., Bharat, S., Chandy, S., Wrubel, J., et al. (2008). HIV-related stigma: Adapting a theoretical framework for use in India. Social Science \& Medicine, 67(8), 1225-1235. https://doi.org/10.1016/j.socsc imed.2008.05.032.

13. Ogden, J., Nyblade, L. (2005). Common at its core: HIV-related stigma across contexts. Washington, DC: International Center for Research on Women.

14. Van Brakel, W. H. (2006). Measuring health-related stigmaA literature review. Psychology, Health \& Medicine, 11(3), 307-334. https://doi.org/10.1080/13548500600595160.

15. Holzemer, W. L., Makoae, L. N., Greeff, M., Dlamini, P. S., Kohi, T. W., Chirwa, M. L., et al. (2009). Measuring HIV stigma for PLHAs and nurses over time in five African countries. SAHARA: Journal of Social Aspects of HIV/AIDS Research Alliance, 6(2), 76-82.

16. Berger, B. E., Ferrans, C. E., \& Lashley, F. R. (2001). Measuring stigma in people with HIV: Psychometric assessment of the HIV stigma scale. Research in Nursing \& Health, 24(6), $518-529$
17. Jeyaseelan, L., Kumar, S., Mohanraj, R., Rebekah, G., Rao, D., \& Manhart, L. E. (2013). Assessing HIV/AIDS Stigma in South India: Validation and abridgement of the Berger HIV Stigma Scale. AIDS Behavior, 17(1), 434-443. https://doi.org/10.1007/ s10461-011-0128-3.

18. Lindberg, M. H., Wettergren, L., Wiklander, M., Svedhem-Johansson, V., \& Eriksson, L. E. (2014). Psychometric evaluation of the HIV Stigma Scale in a Swedish context. PLoS ONE, 9(12), e114867. https://doi.org/10.1371/journal.pone.0114867.

19. Scott, N. W., Fayers, P. M., Aaronson, N. K., Bottomley, A., de Graeff, A., Groenvold, M., et al. (2010). Differential item functioning (DIF) analyses of health-related quality of life instruments using logistic regression. Health and Quality of Life Outcomes, 8 , 81. https://doi.org/10.1186/1477-7525-8-81.

20. Rao, D., Pryor, J. B., Gaddist, B. W., \& Mayer, R. (2008). Stigma, secrecy, and discrimination: Ethnic/racial differences in the concerns of people living with HIV/AIDS. AIDS Behavior, 12(2), 265-271. https://doi.org/10.1007/s10461-007-9268-x.

21. Buseh, A. G., \& Stevens, P. E. (2006). Constrained but not determined by stigma: Resistance by African American women living with HIV. Women and Health, 44(3), 1-18. https://doi. org/10.1300/J013v44n03_01.

22. Riggs, S. A., Vosvick, M., \& Stallings, S. (2007). Attachment style, stigma and psychological distress among HIV + adults. Journal of Health Psychology, 12(6), 922-936. https://doi. org/10.1177/1359105307082457.

23. Emlet, C. A. (2007). Experiences of stigma in older adults living with HIV/AIDS: A mixed-methods analysis. AIDS Patient Care STDS, 21(10), 740-752. https://doi.org/10.1089/apc.2007.0010.

24. Rao, D., Andrasik, M., Acharya, X., \& Simoni, J. M. (2013). Internalized stigma among African Americans living with HIV: Preliminary scale development based on qualitative data. In P. Liamputtong (Ed.), Stigma, discrimination and living with HIVIAIDS (pp. 155-167). Dordrecht: Springer.

25. Crane, P. K., Gibbons, L. E., Jolley, L., \& van Belle, G. (2006). Differential item functioning analysis with ordinal logistic regression techniques-DIFdetect and difwithpar. Medical Care, 44(11), S115-S123. https://doi.org/10.1097/01.mlr.0000245183.28384 .ed.

26. Choi, S. W., Gibbons, L. E., \& Crane, P. K. (2011). lordif: An R package for detecting differential item functioning using iterative hybrid ordinal logistic regression/item response theory and Monte Carlo simulations. Journal of Statistical Software, 39(8), 1-30.

27. R Core Team (2017). R: A language and environment for statistical computing. Vienna: R Foundation for Statistical Computing.

28. Crane, P. K., Gibbons, L., Ocepek-Welikson, K., Cook, K., Cella, D. (2007). A comparison of three sets of criteria for determining the presence of differential item functioning using ordinal logistic regression. Quality of Life Research, 16(Supp 1), 69-84.

29. Bunn, J. Y., Solomon, S. E., Miller, C., \& Forehand, R. (2007). Measurement of stigma in people with HIV: A reexamination of the HIV Stigma Scale. [Research Support, N.I.H., Extramural]. AIDS Education and Prevention, 19(3), 198-208. https://doi. org/10.1521/aeap.2007.19.3.198.

30. Fischer, H. F., Wahl, I., Nolte, S., Liegl, G., Brahler, E., Lowe, B., et al. (2017). Language-related differential item functioning between English and German PROMIS Depression items is negligible. International Journal of Methods in Psychiatric Research, 26(4), e1530. https://doi.org/10.1002/mpr.1530.

31. Brown, T. A., \& Kenny, D. A. (2006). Confirmatory factor analysis for applied research. New York: Guilford Press.

32. Hu, L. T., \& Bentler, P. M. (1999). Cutoff criteria for fit indexes in covariance structure analysis: Conventional criteria versus new alternatives. Structural Equation Modeling-A Multidisciplinary Journal, 6(1), 1-55. https://doi.org/10.1080/107055199095401 18. 
33. Braeken, J., \& van Assen, M. (2017). An empirical Kaiser criterion. Psychological Methods, 22(3), 450-466. https://doi. org/10.1037/met0000074.

34. Chalmers, R. P. (2012). mirt: A multidimensional item response theory package for the R environment. Journal of Statistical Software, 48(6), 1-29.

35. Samejima, F. (1969). Estimation of latent ability using a response pattern of graded scores. Psychometrika, 34(4p2), 1.

36. Hays, R. D., Calderon, J. L., Spritzer, K. L., Reise, S. P., \& Paz, S. H. (2017). Differential item functioning by language on the PROMIS(R) physical functioning items for children and adolescents. Quality of Life Research. https://doi.org/10.1007/s1113 6-017-1691-5.

37. Chadda, R., \& Deb, K. (2013). Indian family systems, collectivistic society and psychotherapy. [Review Article]. Indian Journal of Psychiatry, 55(6), 299-309. https://doi.org/10.4103/00195545.105555.

38. Daun, $\AA$ (1991). Individualism and collectivity among Swedes. Ethnos, 56(3-4), 165-172. https://doi.org/10.1080/00141 844.1991.9981433.

39. Timmerman, M. E., \& Lorenzo-Seva, U. (2011). Dimensionality assessment of ordered polytomous items with parallel analysis.
Psychological Methods, 16(2), 209-220. https://doi.org/10.1037/ a0023353.

40. Teresi, J. A. (2006). Different approaches to differential item functioning in health applications-Advantages, disadvantages and some neglected topics. Medical Care, 44(11), S152-S170. https ://doi.org/10.1097/01.mlr.0000245142.74628.ab.

41. Zumbo, B. (1999). A handbook on the theory and methods of differential item functioning (DIF): Logistic regression modeling as a unitary framework for binary and Likert-type (ordinal) item scores. Ottawa, ON: Directorate of Human Resources Research and Evaluation, Department of National Defense.

42. Jodoin, M. G., \& Gierl, M. J. (2001). Evaluating type I error and power rates using an effect size measure with the logistic regression procedure for DIF detection. Applied Measurement in Education, 14(4), 329-349. https://doi.org/10.1207/S15324818ame140 4_2.

43. Scott, N. W., Fayers, P. M., Aaronson, N. K., Bottomley, A., de Graeff, A., Groenvold, M., et al. (2009). A simulation study provided sample size guidance for differential item functioning (DIF) studies using short scales. Journal of Clinical Epidemiology, 62(3), 288-295. https://doi.org/10.1016/j.jclinepi.2008.06.003.

\section{Affiliations}

\section{Maria Reinius ${ }^{1}$ (1) $\cdot$ Deepa Rao $^{6} \cdot$ Lisa E. Manhart ${ }^{7} \cdot$ Maria Wiklander $^{2} \cdot$ Veronica Svedhem $^{3,5} \cdot$ John $^{\text {Pryor }}{ }^{8}$. Randall Mayer $^{9} \cdot$ Bambi Gaddist $^{10} \cdot$ Shuba Kumar $^{12} \cdot$ Rani Mohanraj $^{12} \cdot$ Lakshmanan Jeyaseelan $^{11}$. Lena Wettergren ${ }^{2,13} \cdot$ Lars E. Eriksson ${ }^{1,3,4}$}

1 Department of Learning, Informatics, Management and Ethics, Karolinska Institutet, SE-171 77 Solna, Sweden

2 Department of Neurobiology, Care Sciences and Society, Karolinska Institutet, SE-141 83 Huddinge, Sweden

3 Department of Infectious Diseases, Karolinska University Hospital, SE-141 86 Huddinge, Sweden

4 School of Health Sciences, City, University of London, London EC1V OHB, UK

5 Department of Medicine, Huddinge, Unit of Infectious Diseases, Karolinska Institutet, 14186 Huddinge, Sweden

6 Department of Global Health and Department of Psychiatry and Behavioral Sciences, Harborview Medical Center, University of Washington, 325 9th Ave, UW Campus Mailbox Number 359931, Seattle, WA 98104, USA
7 Department of Epidemiology, Harborview Medical Center, University of Washington, 325 9th Ave, UW Campus Mailbox Number 35993, Seattle, WA 98104, USA

8 Department of Psychology, Illinois State University, Normal, IL, USA

9 Iowa Department of Public Health, 321 E. 12th St, Des Moines, IA 50319-0075, USA

10 Joseph H. Neal Wellness Center Dba SC HIV Council, 1813 Laurel Street, Columbia, SC 29201, USA

11 Department of Biostatistics, Christian Medical College, Vellore, India

12 Department of Social Sciences, Samarth, Chennai, India

13 Department of Women's and Children's Health, Karolinska Institutet, SE-171 77 Stockholm, Sweden 